\title{
GERMLINE GENETIC TESTING IN PROSTATE CANCER, A POTENTIAL FOR NEW UNDERSTANDING SCREENING, EVALUATION AND TREATMENT OF PROSTATE CANCER
}

\section{MA SALAM}

Germline genetic testing is revolutionizing prostate cancer (PCA) care, with studies revealing inherited mutations (pathogenic variants) in a spectrum of cancer risk genes[1]. Analyses from clinical cohorts of men with PCA have reported germline mutation rates of $15 \%$ to $17 \%$ regardless of stage, with rates of DNA repair mutations reported to be approximately $12 \%$ in men with metastatic PCA[2]. Patients with prostate cancer should now undergo genetic testing of tumor tissue to identify the $30 \%$ or so of patients who can benefit[3]. The highest level of PCA risk has been reported for mutations in BRCA2, and HOXB13, with BRCA2 mutations associated with poor outcomes[1-5].

Genetic testing for men with PCA is being driven increasingly by precision therapy and precision management considerations affecting oncology and urology. For example, olaparib was given Breakthrough Therapy designation by the US Food and Drug Administration for BRCA1/2- or ATM-positive metastatic castration-resistant prostate cancer (mCRPC) on the basis of the TOPARP-A trial demonstrating improved responses particularly in patients with DNA repair mutations[6].

Indeed, mutations in multiple genes (BRCA2, BRCA1, ATM, CHEK2, PALB2, RAD51D, NBN, MLH1, MSH2, PMS2, and MSH6) may provide clinical trial options for men with $\mathrm{mCRPC}$, because genetically informed trials are expanding for PCA treatment [7].

Most experts distinguish three major types of cancer risk. General population risk describes sporadic cancers occurring by chance. In this case, affected patients test negative for any known deleterious mutations within their family, and their close relatives typically do not have the same cancer(s).

Familial cancer risk describes the risk for cancers that arises from both genetic and environmental factors.

www.utfbbd.com
Bangladesh J. Urol. 2019; 22(2): 106-109

These cancers tend to cluster within families and show no specific inheritance pattern.

Hereditary cancers, mutations occur in germ cells and present in every cell in the body. Hereditary cancers occur when a parent passes an altered gene (germline mutation) to a child. Hereditary cancers are often diagnosed at an earlier age than is otherwise typical and affected patients may develop more than one type of cancer. Patients with hereditary cancer(s) often have relatives with the same or related cancers. Only about $5 \%$ to $20 \%$ of cancers are hereditary[8].

Recently, further research has implicated specific mutations in hereditary prostate cancer and has found that men with these variants are at greater risk for highgrade disease. In the Genetic Evaluation of Men study, researchers used multigene sequencing in 200 men who had prostate cancer or were at increased risk. A total of $5.5 \%$ had detectable mutations, which usually involved the DNA repair genes BRCA1, BRCA2, ATM, BRIP1, and MSH6[9].

Patients with prostate cancer should now undergo genetic testing of tumor tissue to identify the $30 \%$ or so of patients, who can benefit - as is already routinely being done for breast, ovarian and lung cancer,

The results come from the phase 3 PROfound study of the PARP inhibitor olaparib (Lynparza, AstraZeneca) in patients who tested positive for DNA repair gene alterations including BRCA1, BRCA2, or ATM mutations.

These were patients with metastatic castration-resistant prostate cancer (mCRPC) whose disease had progressed after treatment with the newer hormonal agents such as abiraterone (Zytiga, Janssen) or enzalutamide (Xtandi, Medivation and Astellas Pharma), and/or with taxane chemotherapy.

In this study, BRCA1 or BRCA2 alterations were seen in $35 \%$ to $40 \%$ of patients, while $18 \%$ to $24 \%$ had ATM 


\section{2(2) 2019}

Germline Genetic Testing In Prostate Cancer, A Potential for New Understanding Screening, Evaluation and Treatment

alterations and $34 \%$ had other alterations. Between $6.6 \%$ and $8.4 \%$ of patients had more than one alteration.

At the Presidential session at ESMO 2019, Maha Hussain, MD, and colleagues presented the initial results of PROfound. Cohort A included patients with alterations in BRCA1, BRCA2 or ATM, while Cohort B patients included any one of 12 other homologous recombination repair alterations (BRIP1, BARD1, CDK12, CHEK1, CHEK2, FANCL, PALB2, PPP2R2A, RAD51B, RAD51C, RAD51D or RAD54L). Patients were randomized $2: 1$ to olaparib (300 $\mathrm{mg}$ bid) or the physician's choice of enzalutamide (160 mg/day) or abiraterone (1000 mg/day + prednisone $5 \mathrm{mg}$ BID). The primary endpoint was radiographic progression-free survival (rPFS) in Cohort A, assessed by blinded independent central review and analyzed via a stratified log-rank test. Crossover to olaparib was allowed after blinded independent central review progression [10].

The results come from the phase 3 PROfound study of the PARP inhibitor olaparib (Lynparza, AstraZeneca) in patients who tested positive for DNA repair gene alterations including BRCA1, BRCA2, or ATM mutations. These were patients with metastatic castration-resistant prostate cancer (mCRPC) whose disease had progressed after treatment with the newer hormonal agents such as abiraterone (Zytiga, Janssen) or enzalutamide (Xtandi, Medivation and Astellas Pharma), and/or with taxane chemotherapy. In this study, BRCA1 or BRCA2 alterations were seen in $35 \%$ to $40 \%$ of patients, while $18 \%$ to $24 \%$ had ATM alterations and $34 \%$ had other alterations. Between $6.6 \%$ and $8.4 \%$ of patients had more than one alteration[11]. The study also indicated a delay in progression seen with olaparib is mpressive because it is considerably higher than the $35 \%$ to $40 \%$ improvements with which been very promising compared to previous prostate cancer studies in this setting[11].

The name "BRCA" is an abbreviation for "BReast CAncer gene." BRCA1 and BRCA2 are two different genes that have been found to impact a person's chances of developing breast cancer. BRCA1 and BRCA2 are tumour suppressor genes and both are inherited in an autosomal dominant fashion with incomplete penetrance. Tumorigenesis in individuals with germline mutations in the BRCA genes requires somatic inactivation of the remaining wild-type allele. Both genes encode large proteins that function in multiple cellular pathways.
Men who carry the BRCA2 gene mutation, which is linked to breast and ovarian cancer, are at risk of developing aggressive prostate cancer and so should undergo regular prostate-specific antigen (PSA) testing, suggest findings from a major study.

The IMPACT Study involved almost 3000 men from 20 countries who were recruited from families known to harbor carriers of mutations of the BRCA1 and BRCA2 genes and who underwent annual PSA testing and biopsy. The results, which were published recently in European Urology, show that men who carried the BRCA2 mutations were almost twice as likely to develop prostate cancer than were noncarriers. They were also diagnosed with prostate cancer at a younger age and had more clinically significant disease[12].

\section{Clinical implications}

Platinum agents induce DNA crosslinks that are substrates for HR DNA repair, which is deficient in BRCA-mutated cells. Therefore, these tumours show high sensitivity to platinum-based chemotherapy, both in vitro ${ }^{96,97,98}$ and in vivo. ${ }^{99,100,101}$ Previous studies in ovarian cancer suggested that mutations in both genes were associated with similar responses to platinum-based chemotherapy. ${ }^{101}$ Recently, Yang et al. ${ }^{102}$ have reported a series of 316 ovarian cancer patients treated with surgery and adjuvant platinumbased chemotherapy in which BRCA2 mutations were associated with improved outcomes, while BRCA1mutated or -hypermethylated tumours were not significantly different from BRCA wild-type cases. BRCA2 mutations were also associated with an increased rate of response to primary platinum chemotherapy.

Platinum agents induce DNA crosslinks that are substrates for HR DNA repair, which is deficient in BRCA-mutated cells. Therefore, these tumours show high sensitivity to platinum-based chemotherapy, both in vitro[13-15]. and in vivo [16-18].Previous studies in ovarian cancer suggested that mutations in both genes were associated with similar responses to platinumbased chemotherapy[18]. Recently, Yang et al[19]have reported a series of 316 ovarian cancer patients treated with surgery and adjuvant platinum-based chemotherapy in which BRCA2 mutations were associated with improved outcomes, while BRCA1mutated or -hypermethylated tumours were not significantly different from BRCA wild-type cases. BRCA2 mutations were also associated with an increased rate of response to primary platinum 
chemotherapy. Interestingly, an increase in genomic instability was found in BRCA2-related tumours, but not in those with BRCA1 loss, due to mutation or hyper methylation. This could indicate that BRCA2 lesions cause more substantial HR defects than BRCA1.

Gallagher et al have recently suggested that those patients with metastatic disease and BRCA mutations do not respond less to the standard chemotherapy regimen with Docetaxel plus prednisolone than $\mathrm{PCa}$ which are BRCA wild type[21].

It was observed that BRCA2 mutations are associated with worse outcome for all survival endpoints, except for survival from metastasis. No difference was seen between mutation carriers and non-carriers, which may be due to a more favourable response to chemotherapy treatments[22].

PARP (Poly(ADP-ribose) polymerases (PARPs) is an enzyme that produces large chains of poly(ADP-ribose) from NAD[23]PARP is involved in the repair of singlestrand DNA breaks and its inhibition produces the accumulation of these lesions which may end in the arrest of the replication fork and the formation of DSBs[24]These DSB are only proficiently repaired by $H R$. In the absence of HR, as occurs when BRCA mutations are present, these DSBs are repaired by error-prone forms of DSB, such as non-homologous en-joining potentially resulting in accumulation of gene aberrations and loss of cell viability[25-26].

Germline mutations in the BRCA genes, mainly in $\mathrm{BRCA} 2$, not only increase the risk of developing $\mathrm{PCa}$, but also have implications in the prognosis and management of the disease. BRCA-related PCa is usually aggressive, and radical treatments are preferred to surveillance, even for low-risk cases.

Further studies are needed to design a tailored management for these patients. An ongoing study, IMPACT, will clarify the benefits of PCa screening in this higher-risk population. Promising clinical trials are evaluating the role of PARP inhibitors in the metastatic setting, but more studies are needed to establish the role of adjuvant treatment, with PARP inhibitors and/or conventional chemotherapy.

The role of chemoprophylaxis in patients with high risk of aggressive forms of $\mathrm{PCa}$ also needs to be addressed. A better characterization of BRCA-related prostate tumours would help to identify sporadic cases with potential lethal forms of the disease that might benefit from the therapeutic strategies designed for BRCA-mutated tumours.

\section{References:}

1. National Cancer Institute: Genetics of prostate cancer (PDQ)-Health professional version. https:/ /www.cancer.gov/types/prostate/hp/prostategenetics-pdq Google Scholar

2. Pritchard CC, Mateo J, Walsh MF, et al: Inherited DNA-repair gene mutations in men with metastatic prostate cancer. N Engl J Med 375:443-453, 2016 Crossref

3. Medline, Google Scholar Medscape Medical News > Conference News > ESMO 2019 'Landmark' Trial in Prostate Cancer With Mutations Liam Davenport October 04, 2019

4. Schrader KA, Cheng DT, Joseph V, et al: Germline variants in targeted tumor sequencing using matched normal DNA. JAMA Oncol 2:104-111, 2016 [Erratum: JAMA Oncol 2:279, 2016] Crossref, Medline, Google Scholar

5. Giri VN, Hegarty SE, Hyatt C, et al: Germline genetic testing for inherited prostate cancer in practice: Implications for genetic testing, precision therapy, and cascade testing. Prostate 79:333339, 2019 Crossref, Medline, Google Scholar

6. Mateo J, Carreira S, Sandhu S, et al: DNA-repair defects and olaparib in metastatic prostate cancer. N Engl J Med 373:1697-1708, 2015 Crossref, Medline, Google Scholar

7. Carlo MI, Giri VN, Antonarakis ES, et al: Evolving intersection between inherited cancer genetics and therapeutic clinical trials in prostate cancer: A white paper from the Germline Genetics Working Group of the Prostate Cancer Clinical Trials Consortium . JCO Precis Oncol https:// doi.org/10.1200/PO.18.00060. [epub ahead of print on August 16, 2018] Google Scholar

8. Walsh PC, Partin AW. Family history facilitates the early diagnosis of prostate carcinoma. Cancer. 1997 Nov;80(9):1871-1874.

9. Giri VN, Obeid E, Gross L, et al. Inherited mutations in men undergoing multigene panel testing for prostate cancer: emerging implications for personalized prostate cancer genetic evaluation JCO Prec Oncol. 2017 May;1:1-17.

10. Zachary Klaassen, MD, MSc - Assistant Professor of Urology, Georgia Cancer Center, Augusta University/Medical College of Georgia Twitter: @zklaassen_md .at the 2019 European 
Germline Genetic Testing In Prostate Cancer, A Pọential for New Understanding Screening, Evaluation and Treatment

Society for Medical Oncology annual meeting, ESMO 2019 \#ESMO19, 27 Sept - 1 Oct 2019 in Barcelona, Spain.

11. Medscape Medical News (C) 2019 Cite this: 'Landmark' Trial in Prostate Cancer With Mutations - Medscape - Oct 04, 2019.

12. Medscape Medical News (c) 2019 Cite this: Men With BRCA2 Should Be Screened for Prostate Cancer - Medscape - Nov 08, 2019.

13. Bhattacharyya A, Ear US, Koller $\mathrm{BH}$, Weichselbaum RR, Bishop DK. The breast cancer susceptibility gene BRCA1 is required for subnuclear assembly of Rad51 and survival following treatment with the DNA cross-linking agent cisplatin. J Biol Chem. 2000;275:23899903. [PubMed ] [Google Scholar]

14. Tassone P, Tagliaferri P, Perricelli A, Blotta S, Quaresima B, et al. BRCA1 expression modulates chemosensitivity of BRCA1-defective HCC1937 human breast cancer cells. $\mathrm{Br} \mathrm{J}$ Cancer. 2003;88:1285-91. [PMC free article ] [PubMed] [Google Scholar]

15. Sakai W, Swisher EM, Karlan BY, Agarwal MK, Higgins J, et al. Secondary mutations as a mechanism of cisplatin resistance in BRCA2mutated cancers. Nature. 2008;451:1116-20. [PMC free article ] [PubMed] [Google Scholar]

16. Byrski T, Gronwald J, Huzarski T, Grzybowska E, Budryk M, et al. Pathologic complete response rates in young women with BRCA1-positive breast cancers after neoadjuvant chemotherapy. J Clin Oncol. 2010;28:375-9. [PubMed ] [Google Scholar]

17. Tan DS, Marchio C, Reis-Filho JS. Hereditary breast cancer: from molecular pathology to tailored therapies. J Clin Pathol. 2008;61:107382. [PubMed ] [Google Scholar]
18. Tan DS, Rothermundt C, Thomas K, Bancroft E, Eeles $\mathrm{R}$, et al. 'BRCAness' syndrome in ovarian cancer: a case-control study describing the clinical features and outcome of patients with epithelial ovarian cancer associated with BRCA1 and BRCA2 mutations. J Clin Oncol. 2008;26:55306. [PubMed] [Google Scholar]

19. Yang D, Khan S, Sun Y, Hess K, Shmulevich I, et al. Association of BRCA1 and BRCA2 mutations with survival, chemotherapy sensitivity, and gene mutator phenotype in patients with ovarian cancer. JAMA. 2011;306:1557-65. [PMC free article] [PubMed] [Google Scholar]

20. Elena Castro, Rosalind Eeles Asian J Androl. 2012 May; 14(3): 409-414. Published online 2012 Apr 23. doi: 10.1038/aja.2011.150 The role of BRCA1 and BRCA2 in prostate cancer

21. Gallagher DJ, Cronin AM, Milowsky MI, Morris MJ, Bhatia J, et al. Germline BRCA mutation does not prevent response to taxane-based therapy for the treatment of castration-resistant prostate cancer. BJU Int. 2011;109:713-9. [PMC free article] [PubMed] [Google Scholar]

22. Castro $\mathrm{E}$, et al. Correlation of germ-line BRCA2 mutations with aggressive prostate cancer and outcome. ASCO Meet Abstr. 2011;29:1517. [Google Scholar]

23. Chambon P, Weill JD, Mandel P. Nicotinamide mononucleotide activation of new DNA-dependent polyadenylic acid synthesizing nuclear enzyme. Biochem Biophys Res Commun. 1963;11:39-43. [PubMed ] [Google Scholar]

24. Ame JC, Spenlehauer C, de Murcia G. The PARP superfamily. Bioessays. 2004;26:882-93. [PubMed] [Google Scholar]

25. Farmer $\mathrm{H}, \mathrm{McC}$ abe $\mathrm{N}$, Lord CJ, Tutt AN, Johnson $\mathrm{DA}$, et al. Targeting the DNA repair defect in BRCA mutant cells as a therapeutic strategy. Nature. 2005;434:917-21. [PubMed] [Google Scholar] 